University of Nebraska - Lincoln

DigitalCommons@University of Nebraska - Lincoln

\title{
A Model for Predicting the Evolution of Damage in Viscoelastic Particle-Reinforced Composites
}

\author{
G. D. Seidel \\ Texas A\&M University, College Station, TX \\ D. H. Allen \\ University of Nebraska-Lincoln, dallen3@unl.edu \\ K. L. E. Helms \\ Intel Corporation \\ S. E. Groves \\ Lawrence Livermore National Laboratory
}

Follow this and additional works at: https://digitalcommons.unl.edu/engineeringmechanicsfacpub

Part of the Mechanical Engineering Commons

Seidel, G. D.; Allen, D. H.; Helms, K. L. E.; and Groves, S. E., "A Model for Predicting the Evolution of Damage in Viscoelastic Particle-Reinforced Composites" (2005). Faculty Publications from the Department of Engineering Mechanics. 54.

https://digitalcommons.unl.edu/engineeringmechanicsfacpub/54

This Article is brought to you for free and open access by the Mechanical \& Materials Engineering, Department of at DigitalCommons@University of Nebraska - Lincoln. It has been accepted for inclusion in Faculty Publications from the Department of Engineering Mechanics by an authorized administrator of DigitalCommons@University of Nebraska - Lincoln. 


\title{
A model for predicting the evolution of damage in viscoelastic particle-reinforced composites
}

\author{
G.D. Seidel ${ }^{\text {a }}$, D.H. Allen ${ }^{\text {b,* }}$, K.L.E. Helms ${ }^{\text {c }}$, S.E. Groves ${ }^{\mathrm{d}}$ \\ a Department of Aerospace Engineering, Texas A\&M University, College Station, TX 77843-3141, USA \\ ${ }^{\mathrm{b}}$ College of Engineering and Technology, University of Nebraska-Lincoln, 114 Othmer Hall, \\ 820 North 16th St., Lincoln, NE 68588-0642, USA \\ ${ }^{\mathrm{c}}$ Thermomechanical Tools and Analysis, Assembly Technology Development, CH5-157, Intel Corporation, \\ 5000 West Chandler Boulevard, Chandler, AZ 85226-3699, USA \\ d Lawrence Livermore National Laboratory, P.O. Box 808, L-342 Livermore, CA 94550, USA
}

Received 3 March 2003; received in revised form 16 December 2003

\begin{abstract}
A viscoelastic cohesive zone model is employed within the framework of a finite element code to analyze a two-phase viscoelastic particle-reinforced composite material consisting of a relatively stiff aggregate embedded in a copolymer binder. The composite of interest, LX17, is noted to have a very large aggregate volume fraction and as such, aggregate grain boundaries were generated within finite element meshes along which viscoelastic cohesive zones have been embedded to model the binder. It has been observed experimentally that the majority of damage in LX17 occurs within the binder, and thus, a damage evolution law has been applied to the viscoelastic cohesive zones that is phenomenological in nature. The responses obtained for the composite from the FEM analysis are then compared to the experimental data compiled by Lawrence Livermore National Labs for various constant strain rate tests conducted by Groves and Cunningham [Tensile and compressive mechanical properties of billet pressed LX17-1 as a function of temperature and strain rate. UCRL-ID-137477. Internal report prepared for Lawrence Livermore National Laboratory, Livermore, CA].
\end{abstract}

Keywords: Cohesive zones; Damage; Fracture; Composites; Viscoelastic; Particle-reinforced; Polycrystalline; Polymer

\section{Introduction}

The plastic bonded explosive (PBX) known as LX17 is a two-phased particle-reinforced composite consisting of irregularly shaped high

\footnotetext{
${ }^{*}$ Corresponding author. Tel.: +1-4024723181; fax: +14024727792.

E-mail address: dhallen@unlnotes.unl.edu (D.H. Allen).
}

explosive (HE) crystals of wet aminated TATB (triamotrinitrobenzene) embedded in a polymeric binder matrix known as KEL-F that consists of a chlorotrifluoroethylene/vinylide fluoride copolymer that is known to degrade with time.

As seen in Fig. 1, the volume fraction of the HE crystals in LX17 is very high at approximately 92.5\%. In addition, Groves et al. (2000) have noted that the mechanical response of LX17 is dominated by the KEL-F binder which has been shown 


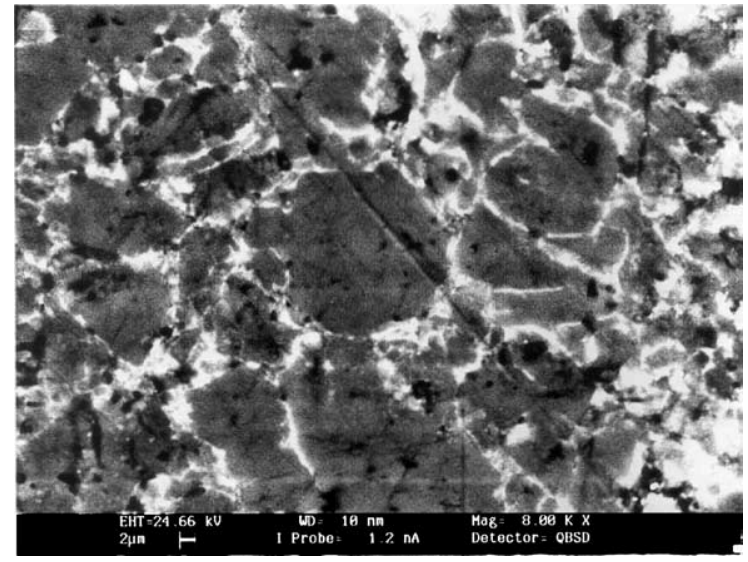

Fig. 1. LX17 at high magnification.

to remain a continuous phase, completely enveloping most crystals. Thus, LX17 is modeled herein with cohesive zones accounting for the KEL-F binder embedded between the HE crystals.

LX17 is of particular interest as it pertains to the certification and lifetime prediction aspects of stockpile stewardship within the United States Department of Energy. Materials such as LX17 have been deployed within the United States stockpile, and due to current political policy, may be required to be in service for up to fifty years. Replacement of LX17 within the stockpile is costly, as is laboratory experimentation. Thus, computational modeling techniques are desired to provide accurate lifetime prediction, thereby allowing for the certification of the stockpile as well as for the coordination of component replacement.

\section{Recent research}

The use of cohesive zones in the study of fracture mechanics is a relatively well-established concept. In an endeavor to remove the stress singularity ahead of the crack tip inherent in the Griffith (1920) theory of classical fracture mechanics, Dugdale (1960) and Barenblatt (1962) were first to propose the use of cohesive zones, or areas ahead of crack tips where non-zero tractions could be specified in such a manner as to inhibit crack tip advancement. While Dugdale proposed a constant traction ahead of the crack tip equal to the yield stress of the surrounding bulk material, Barenblatt introduced the use of non-linear traction-displacement relationships to modify the stress distribution in the region ahead of the crack tip. Both models proved quite successful for modeling ductile fracture in elastic media when compared to experimental observations.

In modeling void nucleation, Needleman (1987) developed a constitutive model for a cohesive zone in which the tractions were described in terms of the normal displacement difference across the cohesive interface in the form of a potential function based on experimental observations and the theoretical potentials used to model atomic bonds. With increasing displacement across the interface, the cohesive forces would increase, reach a maximum, and subsequently vanish as complete decohesion was achieved. Building on Needleman's work, Tvergaard (1990) developed a cohesive zone model with decohesion dependent upon both normal and tangential interfacial displacement differences. Tvergaard utilized a cubic tractiondisplacement law based on a non-dimensional displacement parameter, $\lambda$.

A number of research endeavors have continued to advance the use of cohesive zones in modeling fracture. Gurtin (1979) investigated the use of elastic and viscoelastic cohesive zones in terms of their thermodynamic relationships. Ungsuwarungsri and Knauss (1988) employed non-linear cohesive forces to model crack growth in craze mechanics. For materials that exhibit cohesive or bridging zones at crack tips, Knauss (1993) observed that unstable crack growth could occur before the material had reached a critical value for the crack opening displacement (COD) which became known as subcritical crack growth.

The desire to capture subcritical crack propagation and apparent crack tip (R-) toughening in the modeling of ductile fracture lead to the development of rate and history dependent cohesive zone models. Building on Schapery's (1975a,b,c) work involving discontinuous fracture and cohesive zones, Costanzo and Allen (1993) developed a two-phase cohesive zone model that could be applied to subcritical crack growth using energy methods and observed that void nucleation and 
decohesion ahead of a crack tip. Yoon and Allen (1999) developed a damage evolution law for a non-linear viscoelastic cohesive zone, and Allen and Searcy $(2000,2001 \mathrm{a}, \mathrm{b})$ formulated a micromechanically based non-linear viscoelastic cohesive zone model which was then implemented into an implicit finite element code using a slightly modified version of the Yoon and Allen damage evolution law.

Numerous modeling efforts have focused on the use of cohesive zones for the prediction of the onset and growth of ductile fracture. Rahul-Kumar et al. (2000a) demonstrated the usefulness of cohesive zones in the modeling of peel testing of polymers. Repetto et al. (2000) studied impact loading of glass rods using cohesive zones. Rahul-Kumar et al. (2000b) applied cohesive zones to their study of mixed mode fracture of glass/polymer laminates. Steglich et al. (1999) studied fracture in aluminumtitanium metal matrix composites (MMCs) using cohesive zones. Pandolfi et al. (2000) applied cohesive zones to model a dynamic drop weight test in the study of dynamic fracture of C300 steel. Salvini et al. (2003) employed cohesive zones to model fracture in steel gas pipes.

Focusing specifically on granular composites modeling efforts, Schapery (1986) developed a micromechanical model for particle-reinforced rubber. Clayton (2001) modeled LX17 using linear elastic grains at the appropriate volume fraction embedded in a linear viscoelastic matrix, but without damage evolution incorporated.

Helms et al. (1999) demonstrated the ability to predict grain boundary fracture in polycrystalline solids by employing the Tvergaard (1990) cohesive zone model in an implicit finite element code in studying argillaceous salt. For particle-reinforced composites having large aggregate volume fractions, cohesive zones may be placed along aggregate-to-aggregate boundaries in much the same manner as would be employed for polycrystalline solids making the distinction in terms of modeling between polycrystalline solids and particle-reinforced composites largely negligible. Thus, using a similar approach as Helms, the objective of the present study is to model fracture in high particle volume fraction particle-reinforced composite LX17 making use of the Allen and Searcy (2000, 2001a,b) viscoelastic cohesive zone model with the addition of an internal evolving damage parameter based on the Yoon and Allen (1999) phenomenological damage evolution law in an implicit finite element code. Material parameters for the plastic bonded explosive LX17 are employed in order to compare computational results to experimental results obtained by Groves and Cunningham (2000).

\section{Viscoelastic cohesive zone model and damage evolution law}

A more detailed discussion of the homogenization of the damage zone ahead of a crack tip into the desired traction-displacement relationship is provided by Searcy (1998), and a more detailed development of the incrementalized form of the traction-displacement relationship is provided by Seidel (2002). What follows is a brief summary of those endeavors.

\subsection{Homogenization principle for cohesive zones}

The damaged zone consists of that part of a continuum in the vicinity of a crack tip where local void formation and coalescence create a region of material heterogeneity in the form of load-bearing strands of material known as fibrils, as shown in Figs. 2 and 3. For convenience, a local coordinate system $(n, r, s)$ with base vectors $\hat{e}_{n}, \hat{e}_{r}$, and $\hat{e}_{s}$ has

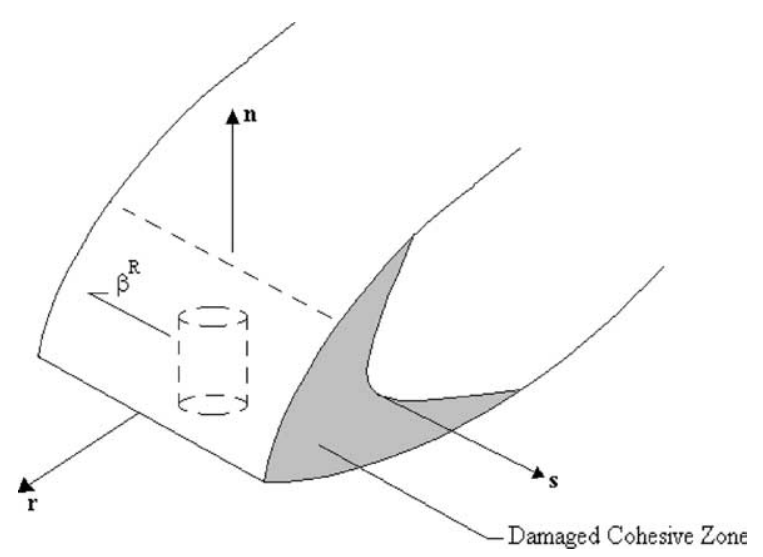

Fig. 2. A general damaged zone. 


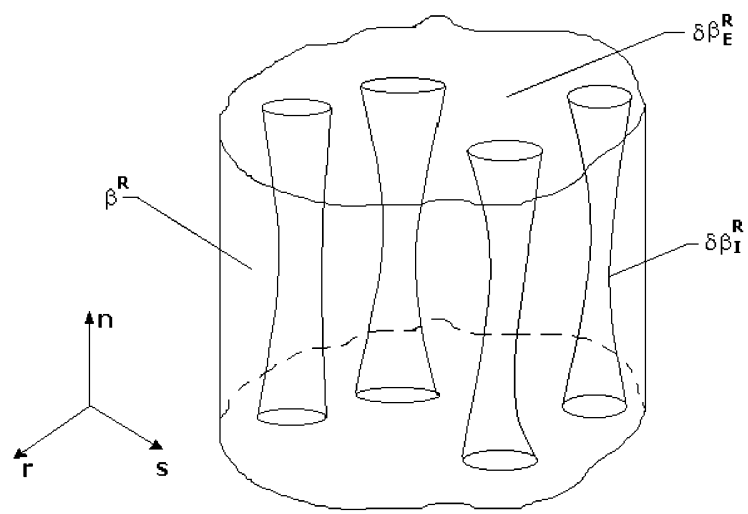

Fig. 3. A representative volume of the damaged zone.

been identified such that $n$ is normal to the plane of the crack tip, $s$ is coincident with the curve of the crack tip, and $r$ is orthogonal to the plane formed by $s$ and $n$.

The principle of homogenization may be applied to the representative volume element (RVE) shown in Fig. 3 assuming that the thickness of the damaged zone and the diameters of the fibrils within the RVE are small relative to other characteristic dimensions of the damaged zone (Searcy, 1998). The homogenization principle states that given the proper geometric constraints, the material properties of a heterogeneous material can be averaged in a manner that will produce an effectively homogeneous material (Christensen, 1979). Applying this principle to the damaged zone allows one to take the material heterogeneity of the damaged zone and create an equivalent area with effectively homogeneous material properties. This leads to the reasonable assumption, essential in formulating analytic solutions, that the damaged zone can be approximated by a mechanically equivalent twodimensional surface of zero volume where cohesive tractions are applied. The resulting model is termed a cohesive zone model (Searcy, 1998).

In order to obtain the homogenized material properties of the damaged zone, the heterogeneity of the damaged zone at the local level of the representative volume must be understood. Thus, in order to formulate the local problem such that a direct micromechanical solution exists, the RVE has been idealized as shown in Fig. 4, where the fibrillated intergranular zone is represented by

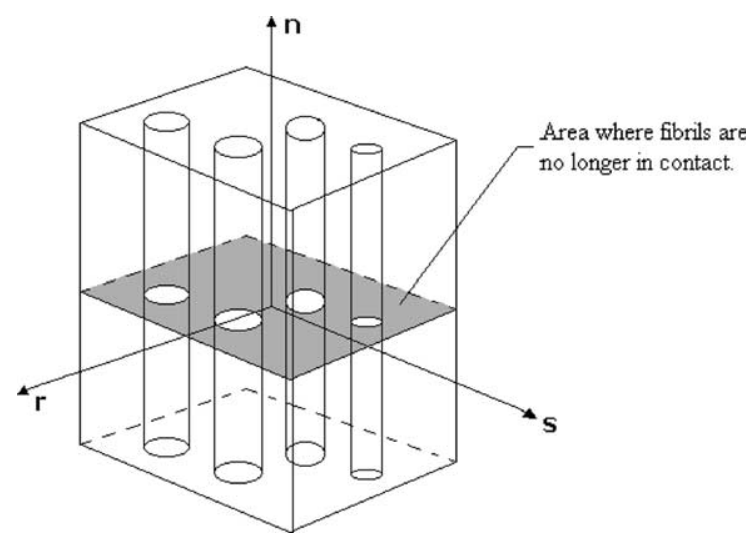

Fig. 4. Defining volume averaged traction in an idealized damaged zone RVE.

right circular prismatic cylinders with linear viscoelastic properties that are assumed to carry only spatially homogeneous uniaxial loads, and where the uniaxial stress-strain relation in a single fibril is given by

$\sigma=\int_{t_{0}}^{t} E^{\mathrm{c}}(t-\tau) \frac{\partial \varepsilon}{\partial \tau} \mathrm{d} \tau$,

where $\sigma$ is the uniaxial stress, $\varepsilon$ is the uniaxial strain, and $E^{\mathrm{c}}$ is the relaxation modulus of the cohesive zone.

Having identified the traction in a single fibril, the area average of the fibril tractions in a representative volume across the cross-sectional area of that representative volume may be expressed by the following

$T=\frac{1}{A} \int_{A} \sigma \mathrm{d} A$,

where $T$ is the area averaged traction.

In the idealized representative volume, the fibrils are all equivalent in length, of the same constitution, and each experience the same uniaxial displacement, thus, the current state of stress in each of the cylinders is uniform. As such, the homogenization of the cohesive tractions can be achieved by evaluating the stress state along the cross-sectional area of the representative volume at the coordinate location $n=0$ as shown in Fig. 4. Thus, one can produce the following expression for the volume averaged traction: 
$T(t)=\left[\sum_{k=1}^{n_{\mathrm{f}}} \frac{A^{k}(t)}{A}\right] \sigma$,

where $A^{k}$ represents the cross-sectional area of the $k$ fibril, $n_{\mathrm{f}}$ is the number of fibrils in the RVE, and $\sigma$ was given in Eq. (3.1). Eq. (3.3) can be further represented by

$T_{i}(t)=\frac{u_{i}(t)}{\delta_{i}} \cdot \frac{(1-\alpha(t))}{\lambda(t)} \cdot\left[\int_{t_{0}}^{t} E^{\mathrm{c}}(t-\tau) \frac{\partial \lambda}{\partial \tau} \mathrm{d} \tau\right]$,

(no sum over $i$ ),

where the subscript $i$ denotes the component of the uniaxial volume averaged traction in the normal and two mutually perpendicular tangential directions allowing for fracture mode-mixity, and where

$\alpha(t) \equiv \frac{A-\sum_{k=1}^{n_{\mathrm{f}}} A^{k}(t)}{A}$,

and

$\lambda=\sqrt{\left(\frac{u_{n}}{\delta_{n}}\right)^{2}+\left(\frac{u_{t}}{\delta_{t}}\right)^{2}+\left(\frac{u_{s}}{\delta_{s}}\right)^{2}}$,

where $\delta_{i}$ are length scale parameters associated with each direction.

The damage parameter, $\alpha$, corresponds to the time-varying area fraction of the growing voids with respect to the cross-sectional area of the representative volume. A phenomenological damage evolution law can be used to express the diminishing fibril volume fraction and is given by

$\dot{\alpha}=c \cdot(1-\alpha)^{m} \lambda^{n}$ for $\dot{\lambda} \geqslant 0$ and $\alpha \leqslant 1$,

$\dot{\alpha}=0$ for $\dot{\lambda} \leqslant 0$ or $\alpha=1$,

where $c, m$ and $n$ are material parameters (Yoon and Allen, 1999). In (3.7a) is an indication of how damage within the cohesive zone will evolve during increasing normalized displacements while (3.7b) indicates that the model will not take into account any damage evolution or crack healing during decreasing normalized displacements. In addition, (3.7b) indicates that macroscale crack growth will occur once the damage reaches a value of $\alpha$ equal to one. As can be seen in Eq. (3.5), this occurs when the number of fibrils in the RVE goes to zero.
It is of interest to point out that the model discussed above is a generalization of those proposed by Needleman (1987) and Tvergaard (1990) used for crystalline metals. However, neither the Needleman nor Tvergaard models included rate dependence, nor did they include a history dependent internal variable associated with damage evolution. In addition, recent research (Williams, 2002) has provided an experimentally based approach for determining the damage evolution law described above.

\subsection{Incrementalization of viscoelastic cohesive} zones

The traction-displacement relationship and damage evolution law may be incrementalized in time by observing that the area averaged traction following a given time increment can be expressed as follows:

$T_{i}(t+\Delta t)=T_{i}(t)+\Delta T_{i}$,

where the subscript $i$ refers to the opening and sliding modes commonly identified in fracture mechanics and thereby denoting the possibility of mode-mixity.

Upon incrementalization, as demonstrated by Allen and Searcy (2000), the following recursive incremental relationship for the non-linear viscoelastic cohesive zone can be expressed in a form conducive to computational algorithms (Seidel, 2002):

$\Delta T_{i}=\frac{(1-\alpha(t+\Delta t))}{\delta_{i}} \cdot\left[E(\Delta t) \cdot \Delta u_{i}\right]+\Delta T_{i}^{\mathrm{R}}$,

(no sum over $i$ ),

where

$$
\begin{aligned}
\Delta T_{i}^{\mathrm{R}}= & \frac{1-\alpha(t)}{\delta_{i}} \cdot\left[-\sum_{j=1}^{P}\left[1-\mathrm{e}^{\frac{-\Delta t}{\rho_{j}}}\right] \sigma_{j}(t)\right] \\
& -\frac{\Delta \alpha}{\delta_{i}} \cdot\left[E_{\infty} \cdot u_{i}(t)+\sum_{j=1}^{P} \sigma_{j}(t)\right] \\
& -\frac{\Delta \alpha}{\delta_{i}} \cdot\left[-\sum_{j=1}^{P}\left[1-\mathrm{e}^{\frac{-\Delta t}{\rho_{j}}}\right] \sigma_{j}(t)\right]
\end{aligned}
$$

(no sum over $i$ ), 
and where

$\Delta u_{i}=u_{i}^{0} \Delta t$,

and

$E(\Delta t)=E_{\infty}+\frac{1}{\Delta t} \sum_{j=1}^{P} \eta_{j}\left(1-\mathrm{e}^{\frac{-\Delta t}{\rho_{j}}}\right)$.

As a result of the incrementalization process there arises a recursive relationship as follows:

$\sigma_{j}(t)=\mathrm{e}^{\frac{-\Delta t}{\rho_{j}}} \cdot\left[\sigma_{j}(t-\Delta t)\right]+\eta_{j} u_{i}^{0}\left[1-\mathrm{e}^{\frac{-\Delta t}{\rho_{j}}}\right]$.

\subsection{Cohesive zones and the finite element method}

Cohesive zones have been implemented within the framework of the finite element method and successfully employed in modeling efforts. Foulk et al. (2000) established an algorithm for the implementation of three dimensional cohesive zones within the framework of the finite element method. In a similar manner, Eqs. (3.9) and (3.10) may be expressed in a form readily implemented into finite element formulations by

$\Delta T_{i}=K_{i} \Delta u_{i}+\Delta T_{i}^{\mathrm{R}}, \quad($ no sum over $i)$,

where $K_{i}$ represents the cohesive zone stiffness,

$K_{i}=\frac{(1-\alpha(t+\Delta t))}{\delta_{i}} \cdot E(\Delta t)$,

and $\Delta T_{i}^{\mathrm{R}}$ represents the residual traction as it was presented in (3.10). Eqs. (3.14) and (3.15) have been implemented into an in-house finite element code to generate the results presented in below.

\section{Material description and characterization}

In modeling polycrystalline solids, cohesive zones can be placed along crystal boundaries for materials where cracking along grain boundaries is observed to dominate the fracture process, as was reported by Helms et al. (1999). In the case of particle-reinforced composites, cohesive zones may be placed between aggregate-to-matrix boundaries when aggregate-matrix debonding or matrix breakdown are observed. However, for particlereinforced composites having large aggregate volume fractions, such as LX17, cohesive zones may be placed along aggregate-to-aggregate boundaries in much the same manner as would be employed for polycrystalline solids.

\subsection{Material characterization}

The binder or matrix material in LX17 is known as KEL-F 800 and is assumed to be isotropic linear viscoelastic in constitution and is accounted for within the cohesive zones due to the high volume fraction of HE crystals $(92.5 \%)$. The HE crystals are modeled as isotropic linear elastic herein using constant strain triangles. Contained in Table 1 are the elastic properties of the HE crystals provided by LLNL (Clayton, 2001).

The viscoelastic properties for the KEL-F binder were provided by LLNL in the form of a 16term Prony series for the shear modulus as a function of time, $G(t)$, obtained from shear tests.

LLNL noted that the Poisson's ratio is time varying for KEL-F, and that the bulk modulus is in fact constant in time. Given the shear modulus, $G(t)$, and the bulk modulus, $K(t)$, one can construct the isotropic linear viscoelastic constitutive relationships in a manner analogous to isotropic linear elastic relationships as follows

$$
\left\{\begin{array}{c}
\bar{\sigma}_{11} \\
\bar{\sigma}_{22} \\
\bar{\sigma}_{33} \\
\bar{\sigma}_{23} \\
\bar{\sigma}_{13} \\
\bar{\sigma}_{12}
\end{array}\right\}=\left[\begin{array}{cccccc}
\widetilde{K}+\frac{4}{3} \widetilde{G} & \widetilde{K}-\frac{2}{3} \widetilde{G} & \widetilde{K}-\frac{2}{3} \widetilde{G} & 0 & 0 & 0 \\
\widetilde{K}-\frac{2}{3} \widetilde{G} & \widetilde{K}+\frac{4}{3} \widetilde{G} & \widetilde{K}-\frac{2}{3} \widetilde{G} & 0 & 0 & 0 \\
\widetilde{K}-\frac{2}{3} \widetilde{G} & \widetilde{K}-\frac{2}{3} \widetilde{G} & \widetilde{K}+\frac{4}{3} \widetilde{G} & 0 & 0 & 0 \\
0 & 0 & 0 & 2 \widetilde{G} & 0 & 0 \\
0 & 0 & 0 & 0 & 2 \widetilde{G} & 0 \\
0 & 0 & 0 & 0 & 0 & 2 \widetilde{G}
\end{array}\right]\left\{\begin{array}{c}
\bar{\varepsilon}_{11} \\
\bar{\varepsilon}_{22} \\
\bar{\varepsilon}_{33} \\
\bar{\varepsilon}_{23} \\
\bar{\varepsilon}_{13} \\
\bar{\varepsilon}_{12}
\end{array}\right\},
$$


Table 1

Isotropic elastic properties for HE crystals

\begin{tabular}{ll}
\hline Young's modulus & $1,430,000 \mathrm{psi}$ \\
Poisson's ratio & 0.33 \\
\hline
\end{tabular}

where the tilde $(\sim)$ denotes the Carson transform, or s-multiplied Laplace transform, and the bar denotes the Laplace transform. Given that the bulk modulus is constant, a direct relationship exists between the shear modulus and the general linear viscoelastic modulus terms, i.e. $C_{i j k l}(t)$, where

$\bar{\sigma}_{i j}=\widetilde{C}_{i j k l} \bar{\varepsilon}_{k l}$.

Now note that the definition of the relaxation modulus is

$E(t) \equiv \frac{\sigma_{11}(t)}{\varepsilon_{11}(t)} \quad$ where $\varepsilon_{11}(t)=\varepsilon_{0} H(t)$

$$
\text { and } \forall \varepsilon_{i j}=0 \text { for } i, j \neq 1 \text {, }
$$

where $\varepsilon_{0}$ is a constant and $H(t)$ is the Heaviside function. Notice that by the above definition, the relaxation modulus is equal to $C_{1111}(t)$ and is therefore directly related to the shear modulus as can be concluded from (4.1) and (4.2) such that

$E(t)=K(t)+\frac{4}{3} G(t)$

Given the above discussion and a bulk modulus of $220 \mathrm{ksi}$, the terms for the Prony series defining $G(t)$ as it is expressed in (4.5) and $E(t)$ as it is expressed in (4.6) are given in Table 2 for the KEL-F binder at $20^{\circ} \mathrm{C}$.

$G(t)=G_{\infty}+\sum_{i=1}^{16} G_{i} \mathrm{e}^{\frac{-t}{\rho_{i}}} \quad$ where $\rho_{i}=\frac{\eta_{S i}}{G_{i}}$,

$E(t)=E_{\infty}+\sum_{i=1}^{16} E_{i} \mathrm{e}^{\frac{-t}{\tau_{i}}} \quad$ where $\tau_{i}=\frac{\eta_{i}}{E_{i}}$

\subsection{Composite homogenization}

Homogenization of a material or composite with a heterogeneous microstructure can be accomplished when the length scale of interest is much larger than the length scale of the material
Table 2

Shear and relaxation Prony terms for KEL-F at $20{ }^{\circ} \mathrm{C}$

\begin{tabular}{lrlrl}
\hline Term & $G(\mathrm{psi})$ & $\rho=\tau(\mathrm{s})$ & $E(\mathrm{psi})$ & $\eta(\mathrm{psis})$ \\
\hline$\infty$ & 0 & N/A & 220,007 & N/A \\
1 & 3,000 & $4.0 \mathrm{E}-08$ & 4,000 & $1.6 \mathrm{E}-04$ \\
2 & 3,168 & $5.0 \mathrm{E}-07$ & 4,224 & $2.122 \mathrm{E}-03$ \\
3 & 5,340 & $5.0 \mathrm{E}-06$ & 7,120 & $3.56 \mathrm{E}-02$ \\
4 & 5,852 & $8.0 \mathrm{E}-05$ & 7,802 & $6.243 \mathrm{E}-01$ \\
5 & 8,440 & $5.58 \mathrm{E}-04$ & 11,253 & 6.27917 \\
6 & 11,200 & $5.80 \mathrm{E}-03$ & 14,933 & $5.8 \mathrm{E}-03$ \\
7 & 13,500 & $6.10 \mathrm{E}-02$ & 18,000 & 86.6114 \\
8 & 15,500 & $5.91 \mathrm{E}-01$ & 20,666 & $12,213.61$ \\
9 & 13,150 & 4.1 & 17,533 & $71,885.3$ \\
10 & 3,205 & 32 & 4,273 & 136,736 \\
11 & 470 & 348 & 627 & 218,196 \\
12 & 61 & $5.0 \mathrm{E}+03$ & 81 & 40,500 \\
13 & 24 & $5.0 \mathrm{E}+04$ & 32 & $1.6 \mathrm{E}+05$ \\
14 & 30 & $5.0 \mathrm{E}+05$ & 40 & $2.0 \mathrm{E}+07$ \\
15 & 25 & $5.0 \mathrm{E}+06$ & 33 & $1.65 \mathrm{E}+08$ \\
16 & 35 & $5.0 \mathrm{E}+07$ & 47 & $2.35 \mathrm{E}+09$ \\
\hline
\end{tabular}

heterogeneity, thereby allowing the material properties to be averaged in a manner so as to produce effectively homogeneous material properties (Christensen, 1979). The geometry used computationally should therefore also exhibit statistically homogeneous material behavior. Voronoi tessellation which uses a random seeding of points to produce a random distribution of polygons of various shapes and sizes that can be used to represent polycrystalline, or granular, materials (Van der Burg and Van der Giessen, 1994) has been employed herein. In terms of homogenization, the Voronoi tessellation must be used to produce a sufficient number of shapes and sizes of grains so that on average, the geometry is homogeneous, i.e. so that including additional grains in the geometry would not change the averaged response of the material. This is done by determining a representative volume element (RVE) for the material.

Defining an RVE is a difficult task as there are a number of manners in which the geometry can introduce statistically inhomogeneous effects. Extensive studies have been performed by the present authors in an endeavor to identify what exactly constitutes an RVE for polycrystalline solids resulting in what is believed to be a minimum number of grains when using a Tvergaard cohesive zone model. Helms (2000) determined 
that a minimum of 500 grains must be included in order to produce statistical homogeneity when damage is included. In the present work several meshes using various numbers of grains are used to make some correlations with the aforementioned work of Helms. However, emphasis is not placed herein on establishing a rigorous definition for an RVE for LX17, since this was previously resolved in the research by Helms (2000).

The grains generated in the Voronoi tessellation correspond to the elastic HE crystals and thus, contain isotropic linear elastic plane stress elements. Adjoining the grains are isotropic linear viscoelastic cohesive zones that correspond to the KEL-F binder matrix and as such constitute crack initiation sites where grain boundary sliding and/ or separating and fracture are allowed.

Shown in Fig. 5 is a sample 10-grain mesh generated using Voronoi tessellation. It should be noted that in Fig. 5, the thick lines denote grain boundaries along which the cohesive zones are

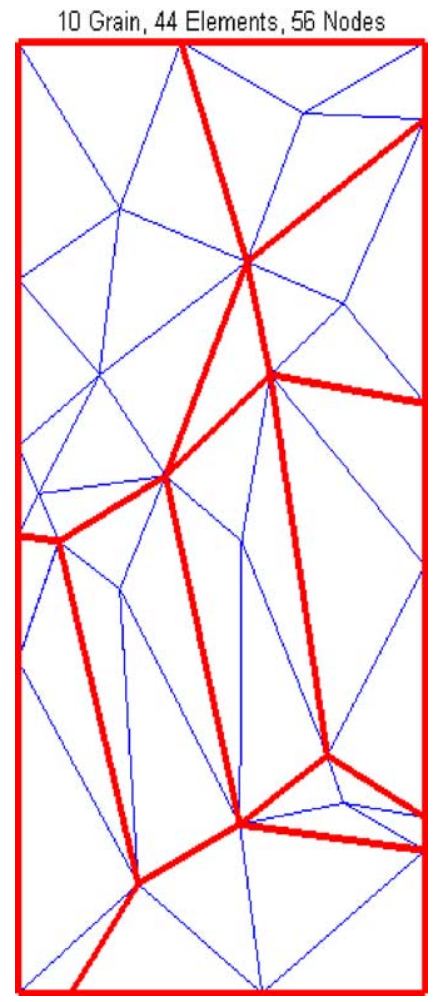

Fig. 5. Ten-grain mesh used in cohesive zone approach. placed while the thin lines identify boundaries between FEM elements contained within a grain connected in a standard finite element sense. Thus, a single grain may be composed of several elements. In fact, the finite element code does not recognize grains directly, but rather, would interpret the mesh shown in Fig. 5 as a collection of ten objects related to one another via cohesive boundary conditions along collateral elements where equal and opposite forces are imposed.

\subsection{Initial and boundary conditions}

Results presented in this study were obtained for the displacement controlled uniaxial bar problem shown in Fig. 6, which, provided the macroscopic physical test specimen is effectively homogeneous, is representative of any location within the uniaxial test specimen. Displacement functions which were linear in time were applied to the nodes along the upper boundary. The boundary conditions were chosen so as to represent one quarter of an RVE located at some location within a test specimen subjected to uniaxial loading. As such, initial conditions consisted of zero tractions

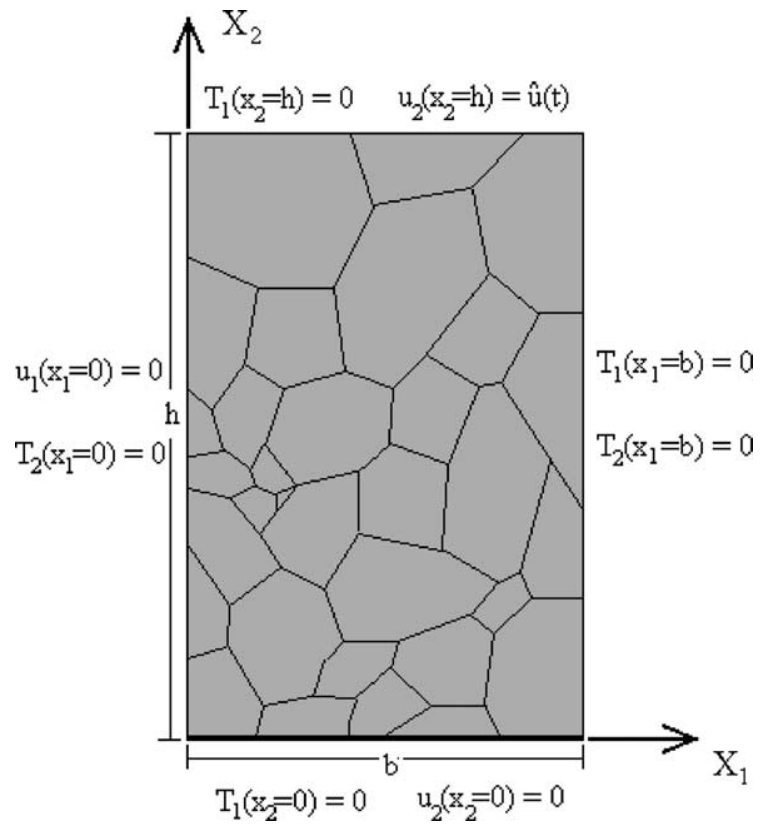

Fig. 6. Uniaxial bar with polygonal elastic grains displayed. 
and displacements along the boundary and zero residual stresses within the matrix.

Boundary conditions were also selected to allow for comparison of computational results with experimental results obtained from LLNL for LX17 subjected to displacement-controlled uniaxial loading. Test specimens used in the LLNL experiments were cylindrical, having a 0.5 in radius and a length of 1 in, and, as Fig. 1 demonstrates, contain grains on the order of microns. Provided the internal composite geometry will allow for homogenization, the specimen may be treated as planar, therefore allowing for a two-dimensional representation of the specimen to be modeled computationally by using plane stress conditions where the out-of-plane stresses are negligible.

\section{Results}

This section presents results using the model. Five strain rates were considered corresponding to the five strain rates presented by Groves and Cunningham (2000), namely, 1.24/s, $1 \mathrm{E}-2 / \mathrm{s}$, $1 \mathrm{E}-4 / \mathrm{s}, 1.25 \mathrm{E}-5 / \mathrm{s}$ and $1.67 \mathrm{E}-6 / \mathrm{s}$. Validation results for the code, GDSFEM, used to generate the results are provided by Seidel (2002), as is a discussion on the implementation of cohesive zone models within the finite element framework and figures of the geometries for all meshes studied.

\subsection{Individual strain rate comparisons for LX17}

Results for a 30-grain mesh are provided and compared with the experimental data compiled by LLNL for constant strain rate tests conducted by Groves and Cunningham (2000) at $20{ }^{\circ} \mathrm{C}$. Three strain rates were considered for comparison, namely, $1.24 / \mathrm{s}, 1 \mathrm{E}-2 / \mathrm{s}$, and $1 \mathrm{E}-4 / \mathrm{s}$. Length scales, $\delta_{i}$, were set to a value of $3.4 \mathrm{E}-4$ in with damage parameters $c, m$, and $n$ from Eq. (3.7a) set to $7.1811,0.82$, and 0.31 , respectively.

Fig. 7 provides the results for the 30-grain mesh for the strain rate of $1.24 / \mathrm{s}$. The dotted lines that demonstrate substantial initial undulation correspond to the experimental data recorded by Groves and Cunningham (2000). The results obtained in the present study are denoted in the

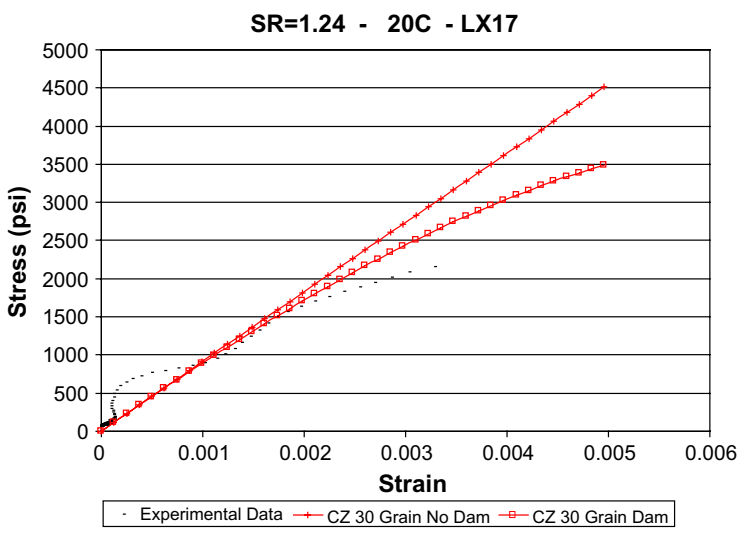

Fig. 7. Thirty-grain LX17 results for strain rate of 1.24/s.

legend with the label "CZ" indicating the use of cohesive zones. The computational results both with and without damage included are represented in the two curves which pass through the experimental data undulation. The damage dependent predictions are significantly closer to the experimental results than the predictions that do not include damage.

The importance in matching the initial stiffness of the experimental data is centered on the observation that the initial experimental test specimen is undamaged. Capturing all of the mechanisms responsible for the evolution of damage as the specimen is loaded is difficult, especially when modeling damage evolution with a phenomenological approach. As such, the initial experimentally obtained stress-strain response of the composite provides the only means of validating the undamaged computational response.

Fig. 8 shows results for the 30-grain mesh for the strain rate of $0.01 / \mathrm{s}$. Many of the same trends as were noticed for the $1.24 / \mathrm{s}$ strain rate results are again noticed in Fig. 8; the undamaged response matches the initial stiffness well and the results with damage included demonstrate a trend similar to that of the experimental results.

Fig. 9 provides the damage versus strain for the strain rate of $0.01 / \mathrm{s}$. In addition, the stress normalized relative to the maximum stress obtained without damage versus strain for both the undamaged and damaged responses are included in the figure. 


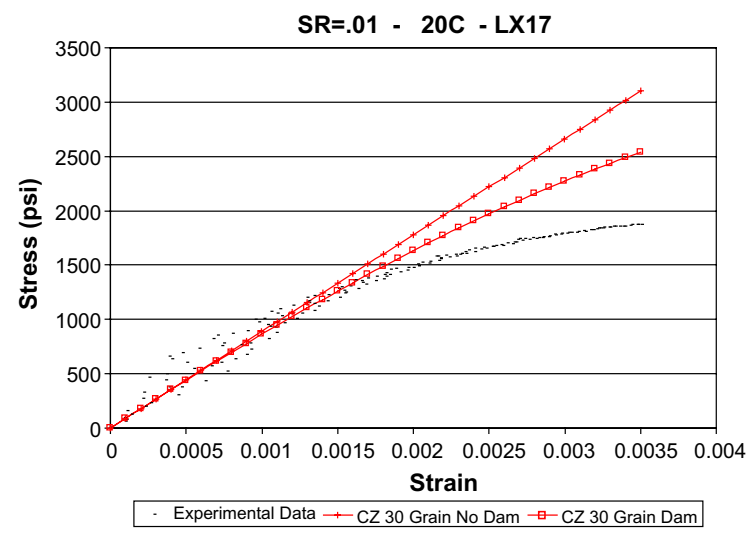

Fig. 8. Thirty-grain LX17 results for strain rate of $0.01 / \mathrm{s}$.

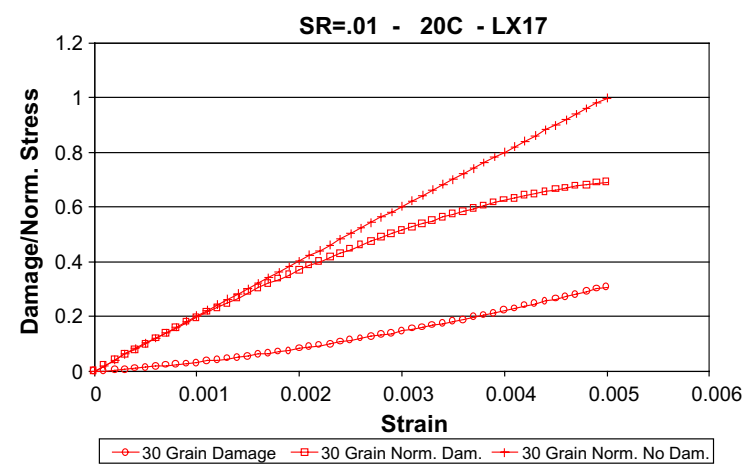

Fig. 9. Thirty-grain LX17 damage results for strain rate of $0.01 / \mathrm{s}$.

Stress-strain results for the strain rate of 1 E-4/s are provided in Fig. 10. Again similar trends are observed as in Figs. 7 and 8, and the experimental data shows substantially less scatter evident in the data.

\subsection{Mesh grain size comparisons}

This subsection provides a brief comparison of the cohesive zone FEM results for LX17 for several meshes, namely, a 5-grain mesh, a 10-grain mesh, a 12-grain mesh, a 30-grain mesh, a 35-grain mesh, a 100-grain mesh, and a 506-grain mesh, all for the single strain rate of $0.01 / \mathrm{s}$ and all with same mesh dimensions. In Fig. 11 is provided relative comparisons of the stress-strain curves for all se-

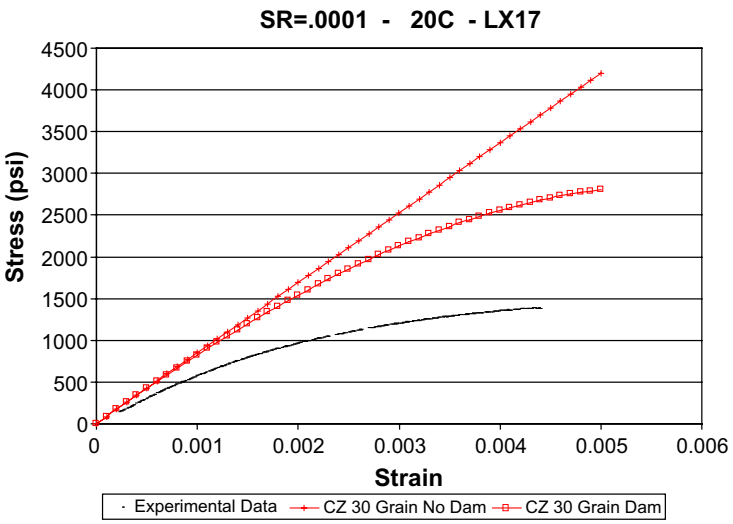

Fig. 10. Thirty-grain LX17 results for strain rate of 1 E-4/s.

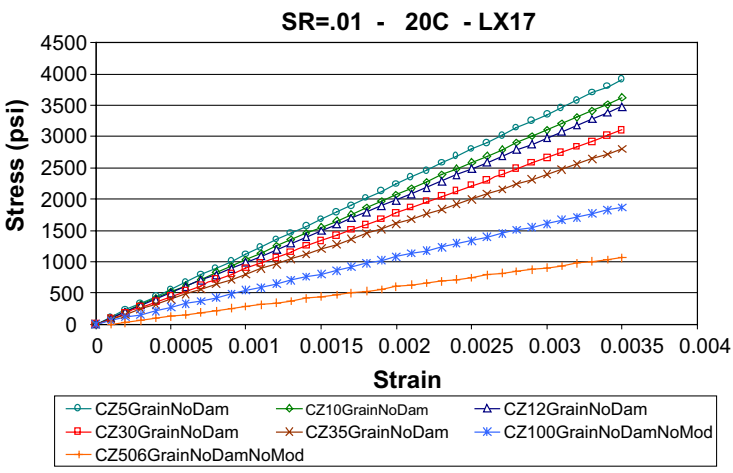

Fig. 11. Mesh comparisons for LX17 for $0.01 / \mathrm{s}$ strain rate w/ out damage.

ven meshes without damage included. The trend observed is consistent with anticipated results, as the stiffness is reduced with increasing number of grains. Careful inspection of the 506-grain mesh results reveals anomalous behavior initially as compared to the other mesh results. This is due to the larger number of degrees of freedom in the 506-grain mesh which made necessary the use of larger time steps in order to complete the simulation in a reasonable amount of time.

Helms (2000) noted for Tvergaard cohesive zones that geometry effects can be considered negligible, i.e. the mesh may be considered a homogenized RVE, when a polycrystalline mesh includes at least thirty grains and that coarse grain meshes are sufficient for simulating mechanical loading before damage is introduced. However, it 
is clear from Fig. 11 that neither the 30-grain nor the 100-grain meshes would constitute a homogenized RVE for LX17 when using viscoelastic

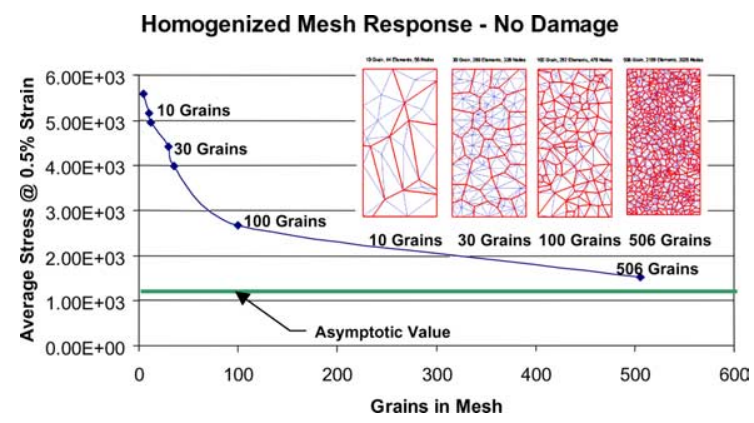

Fig. 12. Undamaged homogenized mesh response.

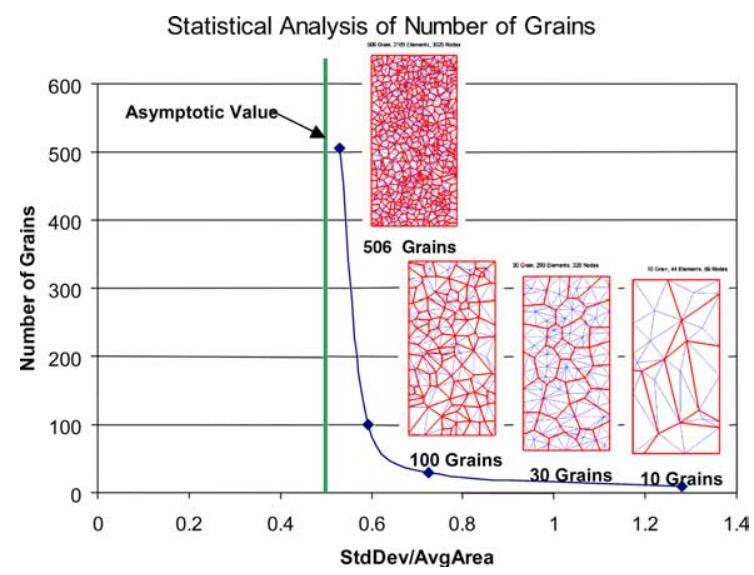

Fig. 13. Geometric prediction of number of grains needed for RVE.

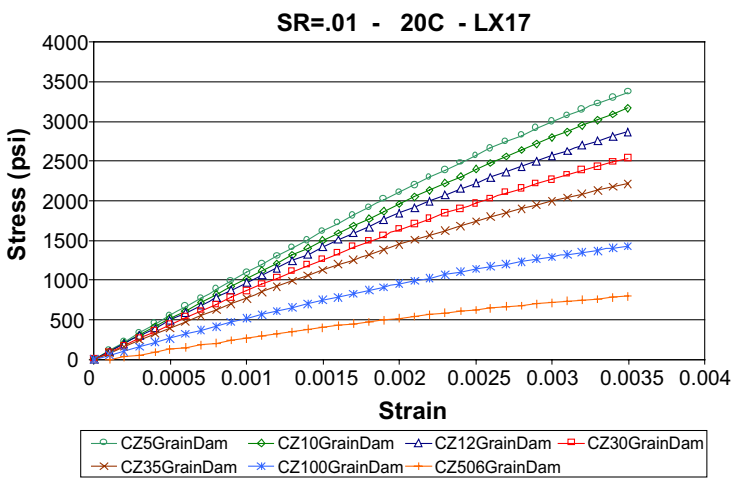

Fig. 14. Mesh comparisons for LX17 for $0.01 / \mathrm{s}$ strain rate w/ damage. cohesive zones. What is not in clear in Fig. 11 is whether or not an RVE for computationally modeling LX17 is being approached, and if it so, how far from it is the 30 -grain mesh. As such,

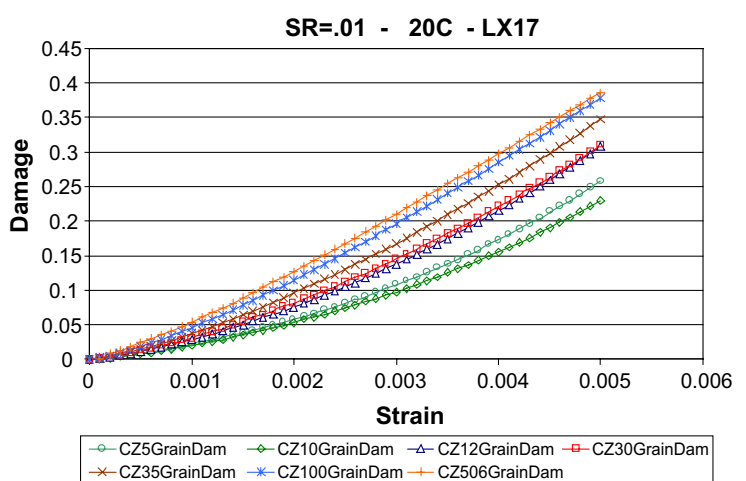

Fig. 15. Mesh damage comparisons for LX17 for $0.01 / \mathrm{s}$ strain rate.

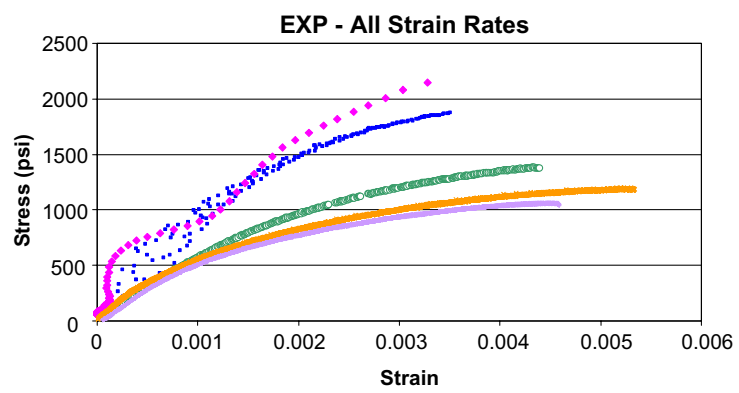

-EXP SR01 •EXP SR1_24 EXP SR0001 × EXP SR1_25E-5 +EXP SR1_67E-6

Fig. 16. Experimental results for all strain rates.

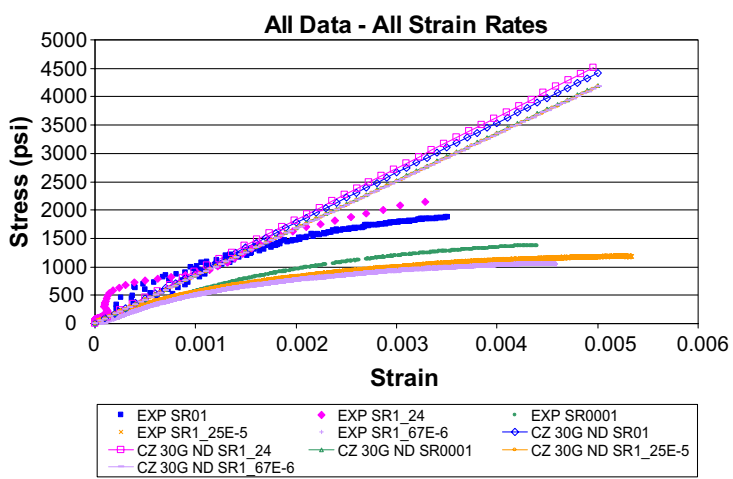

Fig. 17. Experimental and cohesive zone w/out damage FEM results for all strain rates. 
(a) 30 Grain SR 01 Damage Real Geom

Time $=$

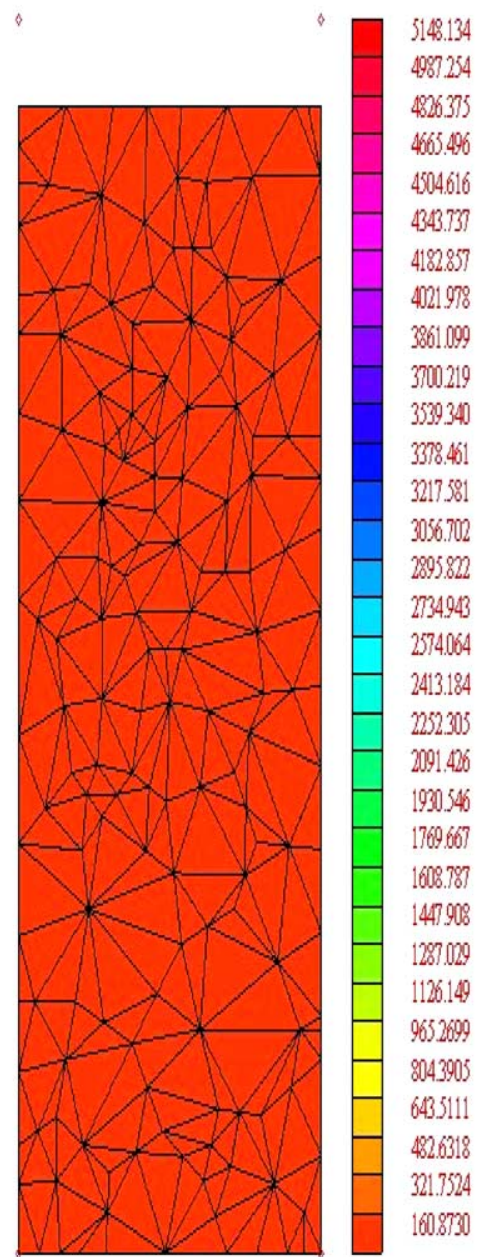

(b) 30 Grain SR 01 Damage Real Geom

Time $=$ (c) 30 Grain SR 01 Damage Real Geom

Time $=$

1900000
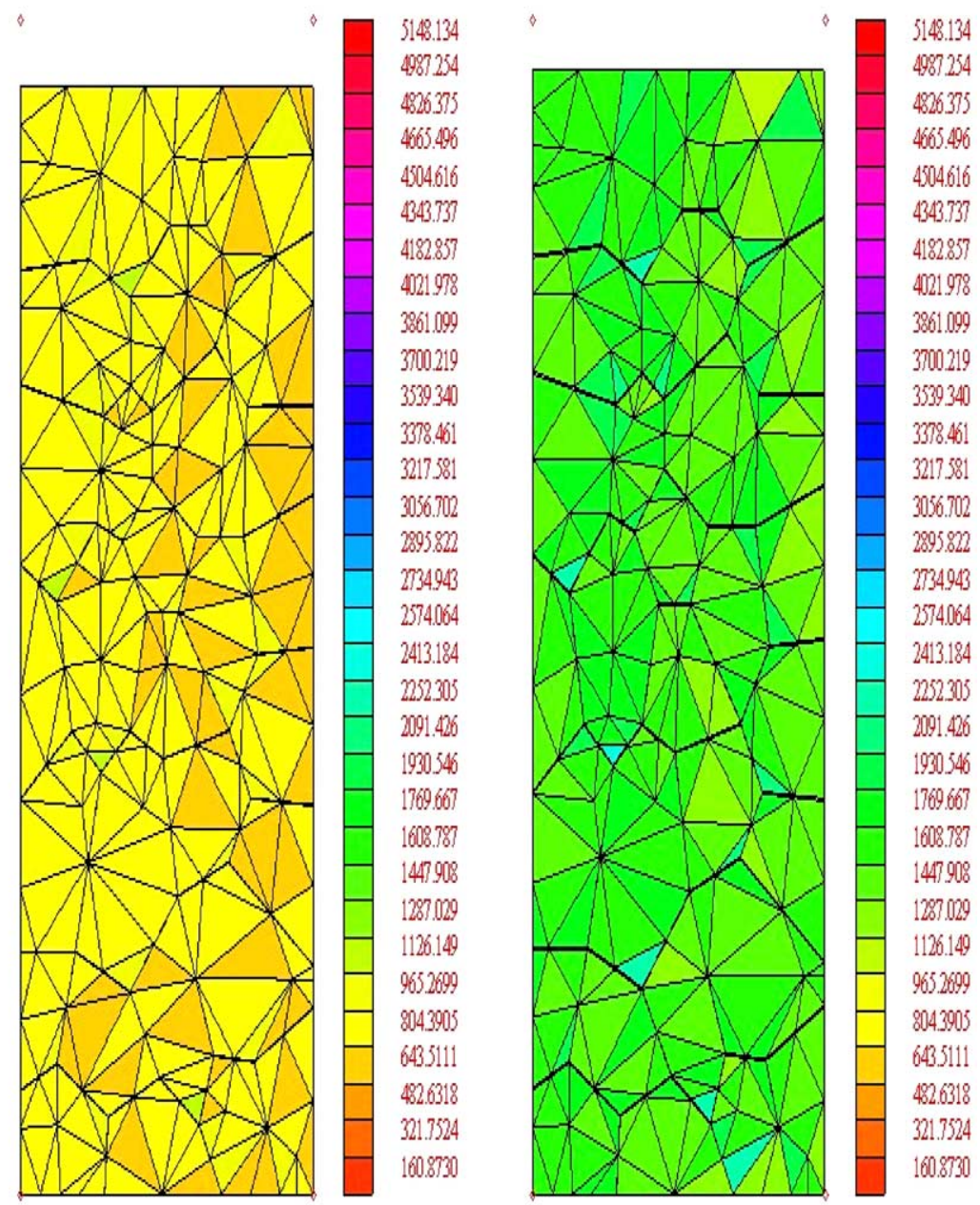

Fig. 18. (a) First frame of 30-grain mesh with damage result for LX17. (b) Second frame of 30-grain mesh with damage result for LX17. (c) Third frame of 30-grain mesh with damage result for LX17. (d) Fourth frame of 30-grain mesh with damage result for LX17. (e) Fifth frame of 30-grain mesh with damage result for LX17. (f) Sixth frame of 30-grain mesh with damage result for LX17.

Fig. 12 is provided in an endeavor to address both issues.

Fig. 12 depicts the value of the stress at a strain of $0.5 \%$ versus the number of grains in the mesh. A saturation effect in terms of grain size can clearly be observed indicating that geometry effects in terms of grain size and orientation in 506-grain meshes are indeed negligible and as such do constitute a computationally converged RVE for the undamaged case depending on the level of accuracy desired. Incorporation of increasingly larger number of grains is hypothesized to asymptotically yield the homogenized value of the stress for the computational RVE. This result appears consis- 
(d) 30 Grain SR 01 Damaye Real Geom

Time $=$

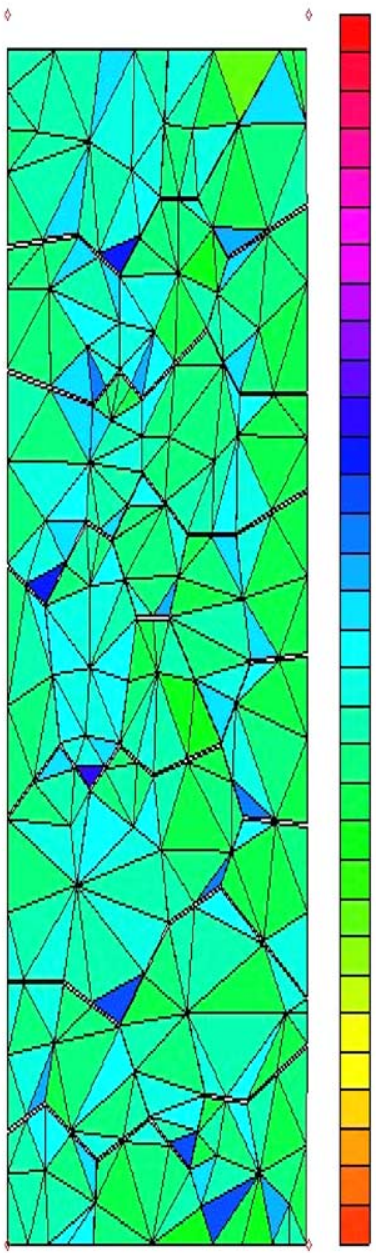

(e)

$\operatorname{Time}=$ (f)

30 Grain SR 01 Damage Real Geom

Time $=$

.400000

\subsection{4 \\ 4226375 \\ 4665.490 \\ 40046.66 \\ 434.3737 \\ 4122.857 \\ 421.978 \\ 3861.099 \\ 300219 \\ 3399340 \\ 3370.461. \\ 321758. \\ 3066.02 \\ 2095822 \\ 2734943 \\ 2574064 \\ 2413.184 \\ 2232305 \\ 2091.425 \\ 1900340 \\ 17696607 \\ 1008.787 \\ 147900 \\ 1287.029 \\ 1126.149 \\ 9652609 \\ 8043905 \\ 6435111 \\ 4226318 \\ 321.7324 \\ 100.8730}
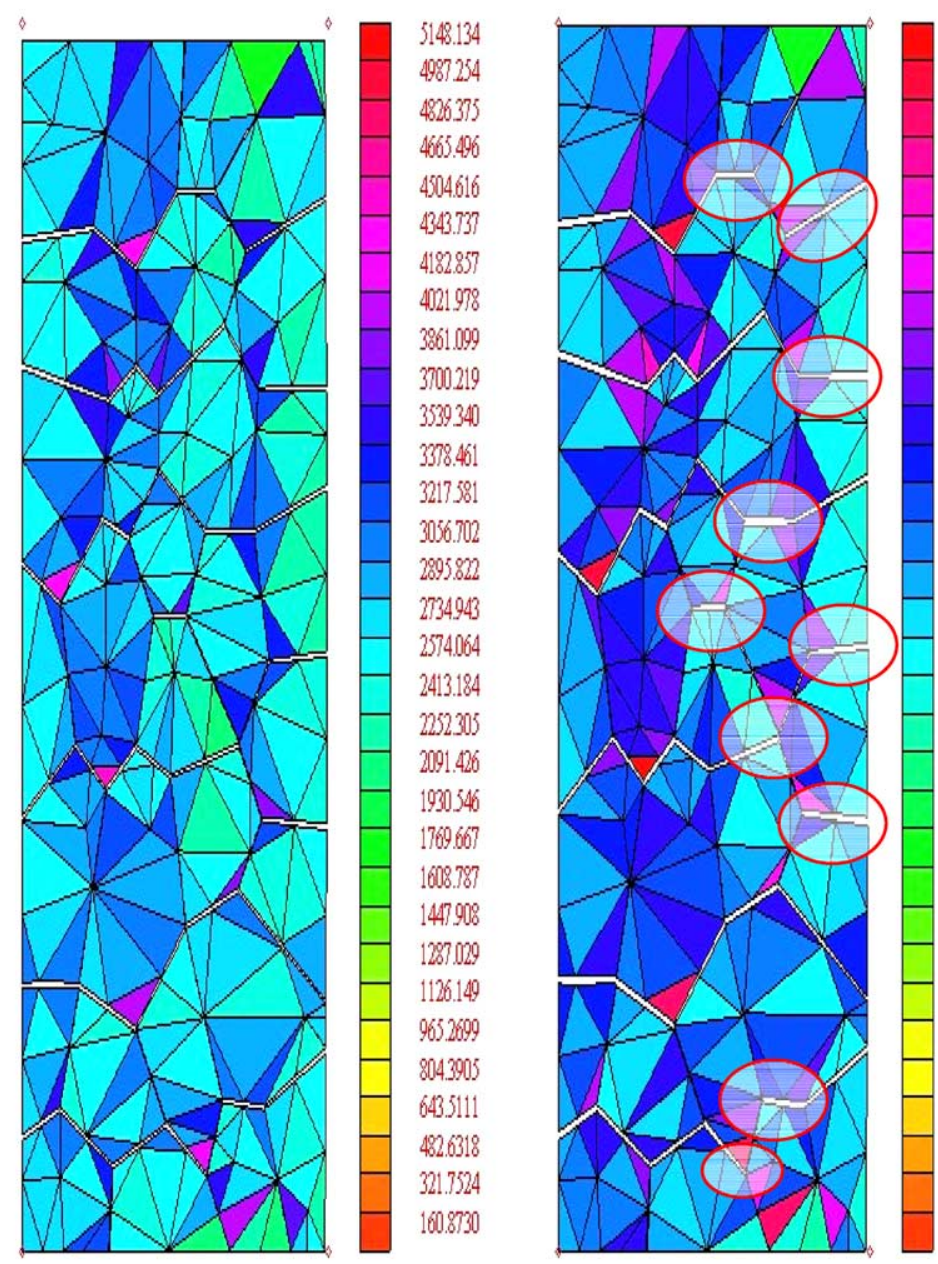

5148.134

4987254

4226375

4665.496

4504616

434.737

$4182.85 ?$

4021978

3861.099

3700219

3339.340

3378.461

3217581

3056.902

2095822

2734943

2574064

2413.184

2232305

2091.426

1930.546

$1760.66 ?$

1608.787

147908

1287.029

1128.149

965.2609

8043905

643.5111

482.6318

321.7324

160.8730

Fig. 18 (continued)

tent with the conclusions made by Helms (2000) where it is stated that statistical consideration of the grain sizes within a given geometry indicate that a value on the order of five hundred grains is sufficient to remove the effects of local geometry on the homogenized response regardless of the material properties of the composite. This is shown in Fig. 13 where it can be seen that the statistical metric of the ratio of standard deviation of grain size to the average grain size begins to demonstrate asymptotic behavior near five hundred grains. Thus, geometric considerations provide a predictive tool for where one may begin to observe homogenized response.

Stress-strain curves for all seven meshes with the damage evolution included are provided in Fig. 14. As all meshes were run at the same strain rate, the damage parameters are identical for all 
seven cases. In the work reported by Helms (2000), a mesh of at least 506 grains was found to be necessary to obtain statistical homogeneity.

Finally, Fig. 15 provides a comparison of the damage versus strain for all seven meshes. The mesh with the most damage at a given strain in Fig. 15 is the 506-grain mesh. This is to be expected, as the total surface area of cohesive zones increases with mesh refinement. Thus, the larger the number of cohesive zones in the mesh, the more the displacements are concentrated in the cohesive zones. Therefore, the KEL-F binder dominates the computational LX17 composite response as was also noted by Groves et al. (2000) in terms of the physical response of the composite.

\subsection{Strain rate to strain rate comparisons}

Fig. 16 demonstrates the experimentally observed stress-strain responses for five strain rates $(1.24 / \mathrm{s}, 1 \mathrm{E}-2 / \mathrm{s}, 1 \mathrm{E}-4 / \mathrm{s}, 1.25 \mathrm{E}-5 / \mathrm{s}$ and $1.67 \mathrm{E}-6 / \mathrm{s})$. As would be expected, the stiffer curves correspond to the higher strain rates and the less stiff curves to the lower strain rates. It is of interest to note the close proximity of the initial stress-strain responses prior to the onset of significant damage. In this range, the experimental results possess similar stiffnesses that appear to be linear and in descending order from highest strain rate to lowest. The slight variance in initial stiffness is believed to be the result of viscoelasticity within the composite. However, the curvature observed in Fig. 16 for each of the strain rates as strain is increased is assumed to be the result of evolving damage.

Also of interest in Fig. 16 is the apparent stronger influence of damage at the slower strain rates. At the slower strain rates, the KEL-F binder in the LX17 composite is significantly more compliant and therefore more susceptible to damage. This effect is captured computationally through the interaction of the Weichert model and the damage evolution law. The dashpots in the Weichert model allow the cohesive zones to displace more at the slower strain rates, thereby allowing more damage to accumulate.

Fig. 17 provides a comparison of experimental and computational results from a 30-grain mesh for all five strain rates where damage has not been included computationally. As was noted in the previous subsection, the results for the largest three strain rates seem to match the initial stiffness of the experimental results rather well, as do the additional two strain rates. In addition, the trend in terms of strain rate effect on initial stiffness as observed in Fig. 16 is repeated for the computational results in Fig. 17. Note that the stress-strain curve demonstrating the highest stiffness corresponds to the highest strain rate and the curve with the lowest stiffness to the slowest strain rate.

\subsection{LX17 animation}

Finally, Fig. 18a-f consist of six frames of an animation of the 30 -grain mesh for the $0.01 / \mathrm{s}$ strain rate with damage evolution included. The contours correspond to the values of the normal stress in the vertical direction. The magnification on the displacements is a factor of fifteen. The apparent cracks in the mesh are actually regions where cohesive zones are opening and sliding and as such, constitute crack initiation sites. With continued loading, many of these crack initiation sites would continue to develop damage and eventually fracture.

Of note in the final frame (Fig. 18f) are the numerous instances where damaged zones consisting of cohesive zones experiencing observable relative displacements appear to terminate in the interior of the mesh as indicated on the figure. This is due to the interaction of the cohesive zones within the mesh, creating preferred paths for damage. These paths along which damage is evolving rapidly are the precursors to macroscale crack propagation and fracture eventually resulting in complete failure of the composite. As such, they represent the loss of statistical homogeneity at the local scale.

\section{Conclusions}

An incrementalized micromechanically-based viscoelastic cohesive zone model with a phenomenological damage evolution law has been implemented into a two-dimensional FEM code in an endeavor to more accurately model the plastic bonded explosive LX17. 
Results have been obtained for a variety of meshes using the material properties and geometry associated with plastic bonded explosive LX17 allowing for comparison of the FEM results to both experimental results for the composite. In comparison to the experimental results, the current approach matched the initial stiffnesses for all strain rates rather well and demonstrated the ability to capture the trends observed in the experimental results due to damage adequately. In addition, an initial effort to study the minimum number of grains necessary to obtain a homogenous mesh response was performed and points to the existence of a computational RVE for the LX17 composite for cases both with and without damage included that is on the order of five hundred grains.

Thus, it is felt that the approach taken in the current work toward modeling LX17 constitutes a successful demonstration of the ability to model ductile fracture in granular composites and high particle volume fraction particle-reinforced composites using viscoelastic cohesive zones and a damage evolution law.

It is concluded that the approach of using viscoelastic cohesive zones to model polycrystalline materials constitutes a significant advancement in modeling ductile fracture. The viscoelastic cohesive zone model and the damage evolution law have been shown to be worthy of continued development and eventual implementation into three-dimensional FEM codes for more advanced simulations.

\section{Acknowledgements}

The authors would like to gratefully acknowledge the support provided by Lawrence Livermore National Labs under contract C02-00139.

\section{References}

Allen, D.H., Searcy, C.R., 2000. Numerical aspects of a micromechanical model of a cohesive zone. Journal of Reinforced Plastics and Composites 19 (3), 240-248.

Allen, D.H., Searcy, C.R., 2001a. A micromechanical model for a viscoelastic cohesive zone. International Journal of Fracture 107, 159-176.
Allen, D.H., Searcy, C.R., 2001b. A micromechanically based model for predicting dynamic damage evolution in ductile polymers. Mechanics of Materials 33, 177-184.

Barenblatt, G.I., 1962. The mathematical theory of equilibrium cracks in brittle fracture. Advances in Applied Mechanics 7, 55-129.

Christensen, R.M., 1979. Mechanics of Composite Materials. John Wiley and Sons, Inc., New York.

Clayton, K.M., 2001. Mechanical modeling of the plastic bonded explosive LX17.M.S. Thesis, Texas A\&M University.

Costanzo, F., Allen, D.H., 1993. A continuum mechanics approach to some problems in subcritical crack propagation. International Journal of Fracture 63, 27-57.

Dugdale, D.S., 1960. Yielding of steel sheets containing slits. Journal of the Mechanics and Physics of Solids 8, 100-104.

Foulk, J.W., Allen, D.H., Helms, K.L.E., 2000. Formulation of a three dimensional cohesive zone model for application to a finite element algorithm. Computation Methods in Applied Mechanics and Engineering 183, 51-60.

Griffith, A.A., 1920. The phenomena of rupture and flow in solids. Philosophical Transactions of the Royal Society of London A 221, 163-197.

Groves, S., Cunningham, B., 2000. Tensile and compressive mechanical properties of billet pressed LX17-1 as a function of temperature and strain rate. UCRL-ID-137477. Internal report prepared for Lawrence Livermore National Laboratory, Livermore, CA.

Groves, S., DeTeresa, S., Cunningham, B., Ciarlo, D., Allen, D.H., Clayton, K., Yoon, C., 2000. Micromechanical characterization tools for highly-filled polymers. UCRLID-137693. Internal report prepared for Lawrence Livermore National Laboratory, Livermore, CA.

Gurtin, M.E., 1979. Thermodynamics and the Griffith criterion for brittle fracture. International Journal of Solids and Structures 15, 553-560.

Helms, K.L.E., Allen, D.H., Hurtado, L.D., 1999. A model for predicting grain boundary cracking in polycrystalline viscoplastic materials including scale effects. International Journal of Fracture 95, 175-194.

Helms, K.L.E., 2000. Modeling the mechanical response and damage evolution in inelastic polycrystalline solids. Ph.D. Dissertation, Texas A\&M University.

Knauss, W.G., 1993. Time dependent fracture and cohesive zones. Journal of Engineering Materials and Technology 115, 263-267.

Needleman, A., 1987. A continuum model for void nucleation by inclusion debonding. Journal of Applied Mechanics 54, 525-531.

Pandolfi, A., Guduru, P.R., Ortiz, M., Rosakis, A.J., 2000. Three dimensional cohesive-element analysis and experiments of dynamic fracture in C300 steel. International Journal of Solids and Structures 37, 3733-3760.

Rahul-Kumar, P., Jagota, A., Bennison, S.J., Saigal, S., 2000a. Cohesive element modeling of viscoelastic fracture: application to peel testing of polymers. International Journal of Solids and Structures 37, 1873-1897. 
Rahul-Kumar, P., Jagota, A., Bennison, S.J., Saigal, S., 2000 b. Interfacial failures in a compressive shear strength test of glass/polymer laminates. International Journal of Solids and Structures 37, 7281-7305.

Repetto, E.A., Radovitzky, R., Ortiz, M., 2000. Finite element simulation of dynamic fracture and fragmentation of glass rods. Computer Methods in Applied Mechanics and Engineering 183, 3-14.

Salvini, P., Fonzo, A., Mannucci, G., 2003. Identification of CTOA and fracture process parameters by drop weight test and finite element simulation. Engineering Fracture Mechanics 70, 553-566.

Schapery, R.A., 1975a. A theory of crack initiation and growth in viscoelastic media: Part I. International Journal of Fracture 11 (1), 141-159.

Schapery, R.A., 1975b. A theory of crack initiation and growth in viscoelastic media: Part II. International Journal of Fracture 11 (3), 369-387.

Schapery, R.A., 1975c. A theory of crack initiation and growth in viscoelastic media: Part III. International Journal of Fracture 11 (4), 549-562.

Schapery, R.A., 1986. A micromechanical model for non-linear viscoelastic behavior of particle-reinforced rubber with distributed damage. Engineering Fracture Mechanics 25 (5/6), 845-867.

Searcy, C.R., 1998. A micromechanical model for a viscoelastic cohesive zone, M.S. Thesis, Texas A\&M University.
Seidel, G.D., 2002. Mechanical modeling of the evolution of damage in the plastic bonded explosive LX17 using viscoelastic cohesive zones, M.S. Thesis, Texas A\&M University.

Steglich, D., Siegmund, T., Brocks, W., 1999. Micromechanical modeling of damage due to particle cracking in reinforced metals. Computational Materials Science 16, 404 413.

Tvergaard, V., 1990. Effect of fiber debonding in a whiskerreinforced metal. Materials Science and Engineering A: Structural Materials: Properties, Microstructure, and Processing A 125 (2), 203-213.

Ungsuwarungsri, T., Knauss, W.G., 1988. A nonlinear analysis of an equilibrium craze: Part I-Problem formulation and solution. Journal of Applied Mechanics 55, 44-51.

Van der Burg, M.W.D., Van der Giessen, E., 1994. Simulation of microcrack propagation in creeping polycrystals due to diffusive grain boundary cavitation. Applied Mechanics Review 47, 122-131.

Williams, J.J., 2002. Two experiments for measuring specific viscoelastic cohesive zone parameters, M.S. Thesis, Texas A\&M University.

Yoon, C., Allen, D.H., 1999. Damage dependent constitutive behavior and energy release rate for a cohesive zone in a thermoviscoelastic solid. International Journal of Fracture $96,55-74$. 